\title{
PROPAGATION CHARACTERISTICS OF THE DES
}

\author{
Marc Davio ${ }^{1,3}$, Yvo Desmedt ${ }^{2}$ and Jean-Jacques Quisquater ${ }^{1}$ \\ 1 Philips Research Laboratory, \\ Avenue Van Becelaere, 2, \\ B-1170 Brussels, Belgium; \\ ${ }^{2}$ Katholieke Universiteit Leuven, \\ Laboratorium ESAT, \\ Kardinaal Mercierlaan, 94, \\ B-3030 Heverlee, Belgium; \\ 3 Université Catholique de Louvain, \\ Batiment Maxwell, \\ Place du Levant, 3 , \\ B-1348 Louvain-la-Neuve, Belgium.
}

Abstract. New general properties in the $S$-boxes were found. Techniques and theorems are presented which allow to evaluate the non-substitution effect in $f$ and the key clustering in DES. Examples are given. Its importance related to the security of DES is discussed. 


\section{Introduction}

The Data Encryption Standard, in short DES, is NBS' cryptographic standard for the protection of commercial computer data (FIPS, 1977). Since 1981, it is also an ANSI standard. In the meantime, it is called DEA by ANSI (ANSI, 1980), and it is yet in use in many industrial applications. Recently it has been proposed to become an ISO (International Standard Organisation) standard under the name of DEA1 (ISO, 1983).

There exist several reasons to explore the internal structure and the functional properties in the DES.

1. It can help to understand the DES. Remark that the design criteria of the DES are still classified (Bernhard, 1982).

2. A better understanding of DES can have two consequences: on the one hand, the detection of weaknesses can speed up a cryptanalysis attack. The detection of inherent strengths will on the other hand simplify the task of defining new standards when they will be needed.

3. The structure can be used in order to simplify or to speed up hardware and software implementations.

To achieve the proposed goals, we first overview (Section 2) the technical description of the DES as it appeared in the NBS publication. The reader, who knows the NBS description of the DES, can skip Section 2. As the full description of all functions in the DES is very long, we refer to the literature (FIPS, 1977; Konheim, 1981; Meyer \& Matyas, 1982; Morris \& al., 1977) for these functions.

In Section 3 general properties in the $S$-boxes and in the key scheduling will be combined.

We analyze several functions in order to combine their properties. As a consequence this can be used to find different cleartexts for which the function $f$ in DES gives the same output. These results can also be used to analyze the key clustering in DES. It means to verify if there exists different keys which gave for most cleartext the same ciphertext.

\section{NBS description of the DES}

The DES algorithm, as described by NBS (FIPS, 1977), consists of three fundamental parts: enciphering computation, calculation of $f(R, K)$ and key schedule calculation. They are are briefly described below.

First observe that several boxes are used in the DES algorithm. It would be a too long explanation to give the details of all these boxes; it can be found in the NBS description. The kind of boxes (e.g. permutation) will be mentioned. 
Remark that the input numbering starts from 0 for some boxes and from 1 for the other ones.

In the enciphering computation, the input is first permuted by a fixed permutation $I P$ from 64 bits into 64 bits. The result is split up into the 32 left bits and the 32 right bits, respectively, this is $L$ and $R$. Then a bitwise modulo 2 sum of the left part $L$ and of $f(R, K)$ is carried out. After this transformation, the left and right 32 bit blocks are interchanged. Observe that the encryption operation continues iteratively for 16 steps or rounds. In the last round, no interchange of the last obtained left and right parts is performed; the output is obtained by applying the inverse of the initial permutation $I P$ to the result of the 16-th round.

In the calculation of $f(R, K)$ the 32 right bits are first expanded to 48 bits in the box $E$, by taking some input bits twice, others only once. Then a bitwise modulo 2 sum of the expanded right bits and of 48 key bits is performed. These 48 key bits are obtained in the key schedule calculation, which will be explained later on. The results of the modulo 2 sum go to the eight $S$-boxes; each of these boxes has six inputs and four outputs. The $S$-boxes are nonlinear functions. The output bits of the $S$-boxes are permuted in the box $P$.

Let us finally describe the key schedule calculation. The key consists of 64 bits, of which 56 bits only are used. The other 8 bits are not used in the algorithm. The selection of the 56 bits is performed in box $P C_{1}$, together with a permutation. The result is split into two 28 bit words $C$ and $D$. To obtain the 48 key bits for each iteration, the words $C$ and $D$ are first left shifted once or twice. A selection and a permutation $P C_{2}$ are then applied to the result. The output of $P C_{2}$ is the 48 bit key word which is used in $f(R, K)$. An additional table tells the user how many shifts must be performed to obtain the next 48 key bits of the key for the following round. DES can be used in four modes (FIPS, 1980; Konheim, 1981).

\section{Propagation characteristics}

We first analyze the new properties, which we observed in the expansion phase, the $S$-boxes and the key scheduling. We combine our results with older ones (Davio \& al., 1983) in order to discuss the non-substitution property in $f$ and the key clustering in DES. Let us first discuss the importance of the fact that $f$ is not a substitution and of the key clustering.

\subsection{The importance of the propagation characteristics}

If $f$ is not a substitution the cardinality of the image play an important role in the evaluation of the security of DES. Indeed if the image of $f$ contains only one element, the DES is completely linear. More generally, if the cardinality of 
the image of $f$ is small DES may be insecure.

If there exist a key clustering in DES, it may be that for a large amount of cleartexts the effect of modifying the key on a special way do not affect the ciphertext. If this is true for DES it simplifies enormously an exhaustive attack.

\subsection{The expansion phase}

The expansion phase plays a very important role in this section.

\subsection{The $S$-boxes}

\subsubsection{An introduction}

We observed several new properties in the $S$-boxes. Most of our new properties are valid for all $S$-boxes and are consequently called "general properties". In the following sections some of these properties are used in order to analyze in which measure $f$ is not a substitution and to analyze the key clustering. We did not apply all general properties in the following sections; perhaps in the future one will be able to explain why the $S$-boxes have these properties or to use them in some deeper analysis of DES.

Two kinds of properties are discussed. In the first kind we fix some input bits of the $S$-boxes $(1,2, \ldots$, or 5 of the 6 possible bits). We are interested in what changes are propagated at the output and how? E.g. for the output one can wonder if the 4 output bits are always be distinct if we change the non-fixed input bits, or if for some inputs the output is not affected. Secondly we discuss how the output changes if we complement some input bits of the $S$-boxes.

We number the inputs of one $S$-box by abcdef. We number the $S$-boxes from 1 to 8 and denote them as $S_{i}$. Remark that representations of the $S$-boxes, other than in the NBS norm, may be useful (Davio \& al., 1983).

\subsubsection{Properties of the $S$-boxes if some input bits are fixed}

The inputs $a, b, e, f$ of the $S$-boxes play a special role in DES. Indeed one half of the message input bits in each round influences two $S$-boxes. These bits will go to the mentioned input bits. These bits will play an important role in the analysis of the non-substitution property of the function $f$ in DES. The next properties draw special attention to the mentioned input bits. The following properties can however easily be generalized. One can easily verify them using a computer program.

We number the properties by a double numbering technique, such that it is easy to refer to them.

1. The observed properties hold for all $S$-boxes. We analyze if the output 
of an $S$-box can or cannot change if one modifies the inputs of an $S$-box in the following way:

(a) fix the inputs $e$ and $f$

(b) one is allowed to change $c$ and $d$ to an arbitrary value $c^{\prime}$ and $d^{\prime}$

(c) one changes the inputs $a$ and $b$ as described in the properties

1.1. $\neg\left(\forall c, d, c^{\prime}, d^{\prime}, e, f: S_{i}(0,0, c, d, e, f) \neq S_{i}\left(1,0, c^{\prime}, d^{\prime}, e, f\right)\right)$

1.2. $\neg\left(\forall c, d, c^{\prime}, d^{\prime}, e, f: S_{i}(0,1, c, d, e, f) \neq S_{i}\left(1,1, c^{\prime}, d^{\prime}, e, f\right)\right)$

1.3. $\forall c, d, c^{\prime}, d^{\prime}, e, f: S_{i}(0,1, c, d, e, f) \neq S_{i}\left(1,0, c^{\prime}, d^{\prime}, e, f\right)$

1.4. $\forall c, d, c^{\prime}, d^{\prime}, e, f: S_{i}(0,0, c, d, e, f) \neq S_{i}\left(1,1, c^{\prime}, d^{\prime}, e, f\right)$

Remark: One can wonder why e.g. $S_{i}(0,0, c, d, e, f)$ was not compared with $S_{i}\left(0,1, c^{\prime}, d^{\prime}, e, f\right)$. This property is already known, indeed it is known (Konheim, 1981) that each row (see NBS notation) of each $S$-box is a permutation. In other words $S_{i}(a, b, c, d, e, f) \neq S_{i}\left(a, b^{\prime}, c^{\prime}, d^{\prime}, e^{\prime}, f\right)$ independent of $b, c, d, e, b^{\prime}, c^{\prime}, d^{\prime}, e^{\prime}$. The properties described here are in fact a generalization of it.

2. The observed properties hold for all $S$-boxes, except property 2.4 . We analyze if the output of an $S$-box can or cannot change if one modifies the inputs of an $S$-box in the following way:

(a) fix the inputs $a$ and $b$

(b) one is allowed to change $c$ and $d$ to an arbitrary value $c^{\prime}$ and $d^{\prime}$

(c) one changes the inputs $e$ and $f$ as described in the properties

2.1. $\neg\left(\forall a, b, c, d, c^{\prime}, d^{\prime}: S_{i}(a, b, c, d, 0,0) \neq S_{i}\left(a, b, c^{\prime}, d^{\prime}, 0,1\right)\right)$

2.2. $\neg\left(\forall a, b, c, d, c^{\prime}, d^{t}: S_{i}(a, b, c, d, 1,0) \neq S_{i}\left(a, b, c^{\prime}, d^{\prime}, 1,1\right)\right\}$

2.3. $\neg\left(\forall a, b, c, d, c^{\prime}, d^{\prime}: S_{i}(a, b, c, d, 0,1) \neq S_{i}\left(a, b, c^{\prime}, d^{\prime}, 1,0\right)\right)$

2.4. If $i \neq 4$ then:

$\neg\left(\forall a, b, c, d, c^{\prime}, d^{\prime}: S_{i}(a, b, c, d, 0,0) \neq S_{i}\left(a, b, c^{\prime}, d^{\prime}, 1,1\right)\right)$

If $i=4$ then:

$\forall a, b, c, d, c^{\prime}, d^{\prime}: S_{i}(a, b, c, d, 0,0) \neq S_{i}\left(a, b, c^{\prime}, d^{\prime}, 1,1\right)$

Remark. The properties 1.3 and 1.4 change if one also allows that the input $e$ changes to the input $e^{\prime}$. Then it will be possible to find identical outputs for special inputs. A similar remark is true for property $2.4(i=4)$ if one allows that the input $b$ changes. 


\subsubsection{Complementation properties of the $S$-boxes}

A well known (Hellman \& al., 1976) property for the $S$-boxes is that if one complements one input of an $S$-box at least two output bits will change. We analyze the effect of complementing two input bits, while leaving the other ones unchanged. It is evident that one can easily generalize our properties for the case that 3 or more bits are complemented. The first aim was to observe whether it is possible to maintain a constant output if only two bits are complemented. First observe that in order to maintain a fixed output one has to complement bit a or $f$, otherwise we conflict with the permutation property of the "rows" in the $S$-boxes. For special abcde $f$ inputs the output of an $S$-box remains unchanged if one complements two of the input bits.

It is remarkable that only if $a b$ is complemented we have that the output for all $S$-boxes changes. This is however very easy to prove starting from our properties 1.3 and 1.4 of the previous section. In a similar way one can use properties 2.3 and 2.4 to prove the above conclusion for complementing $e f$.

\subsection{The key scheduling}

In our analysis of the key clustering we used in detail the key scheduling in DES. The ideas of Neutjens on the key scheduling in DES were very useful in this context (Neutjens, 1983). We now overview them and explain them systematically. We number the 56 key bits from 1 to 64 as in the NBS description (FIPS, 1977).

First of all remark that after $P C_{1}$ one can split up the key scheduling in DES completely in two parts. $P C_{2}$ does not affect this decomposition (Davio \& al., 1983). As a consequence of this decomposition, one can separate for one round in DES the selection of the key bits which will influence the first $4 S$-boxes and the last $4 S$-boxes. Let us now construct the equivalent scheme. All used notations, e.g. the registers $C$ and $D$, originate from the NBS representation of DES.

We represent the register content of $C$ by $\left(c_{1}, c_{2}, \ldots, c_{28}\right)$ and that of $D$ by $\left(d_{1}, d_{2}, \ldots, d_{28}\right)$. Mostly in the key scheduling the registers $C$ and $D$ are shifted twice to obtain the $K_{i}$ of the $i^{\text {th }}$ round, e.g. $\left(c_{1}, c_{2}, c_{3}, \ldots, c_{28}\right)$ is transformed into $\left(c_{3}, c_{4}, c_{5}, \ldots, c_{2}\right)$. This can now be reformulated for the $C$ register as one shift on the following two registers $\left(c_{1}, c_{3}, c_{5}, \ldots, c_{27}\right)$ and $\left(c_{2}, c_{4}, c_{6}, \ldots, c_{28}\right)$. We call them respectively the odd and the even registers. One can then realize the key scheduling with 4 registers instead of two, which shift only once when in the NBS the registers shift two times. This reorganization affects the $P C_{2}$.

One has now still to discuss what happens if only one shift is performed on $C$ and $D$ as in the iterations $1,2,9$ and 16 using our equivalent representation. The first shift in the first iteration can be realized together with $P C_{1}$. In the other situations we interchange the content of the odd and the even registers, by performing first a shift on the old content of the odd register and no shift 
on that of the even register. We then change also the name of each register: odd becomes even, even becomes odd. Indeed $\left(c_{1}, c_{3}, c_{5}, \ldots, c_{27}\right),\left(c_{2}, c_{4}, c_{6}, \ldots\right.$, $\left.c_{28}\right)$ is then changed into $\left(c_{2}, c_{4}, c_{6}, \ldots, c_{28}\right),\left(c_{3}, c_{5}, c_{7}, \ldots, c_{1}\right)$. One can verify that previous operations are identical to one shift in the NBS notation.

The register $D$ can be treated in a similar way. Remark that it is more difficult to perform one shift in the NBS representation. However we are able to see better which bits of the key affect a particular $S$-box.

Let us now apply all the described properties.

\subsection{The function $f$ is not one-to-one for fixed $\mathbf{K}$}

Let us remember here that $f$ consists of the expansion box $E$, of the EXORing with the key bits, of the $S$-boxes and of the permutation $P$. It has sometimes been wondered whether the $f$ function is by itself a substitution. The answer to that question is negative (Davio \& al., 1983; Konheim, 1981). A more systematic discussion is given in this section.

We will now use the properties described in section 3.3.2. to demonstrate how they can be used in the analysis of the non-substitution of $f$. Evidently we assume that the key $K$ is fixed. We analyze which bits of the message part $R$ (see NBS notation) one must change in order to maintain the same output of $f$. We will progressively increase the number of changed bits. First we only change the inputs (or message part of the input) of one, two and then three $S$-boxes and generalize afterwards. We will mostly use the new as well as the well known (Hellman \& al., 1976; Konheim, 1981) general properties of the $S$-boxes, together with the structure of $E$ (Davio \& al., 1983).

Theorem 1: If for fixed key, one only changes the input of one $S$-box the output of $f$ will change.

Proof : In order not to affect the inputs of the other $S$-boxes one can only change the inputs $c$ and $d$. However if $a$ and $f$ are not changed an $S$-box forms a substitution.

$\circ$

Theorem 2: If for fixed key, one changes only the input of two neighbourhood $S$-boxes the output of $f$ will change.

Proof: Let us call the two affected $S$-boxes, $S_{i}$ and $S_{i+1}$ and let us define $S_{9}$ as being $S_{1}$ (this again shows that it can be more interesting to start the numbering from 0, see (Davio \& al., 1983)). In order not to affect the input of $S_{i-1}$ the inputs $a$ and $b$ of $S_{i}$ may not change and similarly for the inputs $e$ and $f$ of $S_{i+1}$ in order not to affect the inputs of $S_{i+2}$. In order not to conflict with the permutation properties of the "rows" of the $S$-boxes and using the previous remark, at least $f$ in $S_{i}$ must be complemented in order to maintain a fixed output. A similar remark is true for the input $a$ of $S_{i+1}$. As consequence of the 
expansion box $E$ a complementation of the input $e$ (respectively $f$ ) of $S_{i}$ is equal to a complementation of the input of a (respectively $b$ ) of $S_{i+1}$. So in order to produce a same output we have at least to complement $a$ and $b$ in $S_{i+1}$. Remark that the inputs $c$ and $d$ in $S_{i+1}$ do not influence the proof. In other words even if one additionally changes the inputs $c$ and $d$ in $S_{i+1}$ or do not, the output of $S_{i+1}$ will change, by virtue of property 1.3 and 1.4 of the $S$-boxes.

o

Theorem $3:$ If for fixed key, one changes only the input of three neighbourhood $S$-boxes the output of $f$ will for some inputs remain identical, only if one complements at least the inputs $a, b$ and $e$ of the middle of the three $S$-boxes, the input $c$ or d of the last $S$-box and if one does not complement the input $f$ of the middle of the three $S$-boxes.

Proof: We call the three $S$-boxes $S_{i-1}, S_{i}$ and $S_{i+1}, S_{0}$ is equal to $S_{8}$ and $S_{9}$ equals $S_{1}$. The proof is for a large part similar to that of theorem 2 . Let us first give the similar part of the proof.

We must fix the inputs $a$ and $b$ of $S_{i-1}$, and $e$ and $f$ of $S_{i+1}$. The input $f$ of $S_{i-1}$ must be complemented and similarly for the input $a$ of $S_{i+1}$. This last condition is equivalent to saying that the inputs $b$ and $e$ of $S_{i}$ must be complemented. Now we apply the consequences of theorem 2 to continue our proof.

If $a$ and $b$ are both complemented in $S_{i+1}$, the output will change (see proof of theorem 2 or properties 1.3 and 1.4 of the $S$-boxes). Using previous observations the input $b$ in $S_{i+1}$ may not be complemented, or equivalently $f$ in $S_{i}$. At this moment we already know that for $S_{i} b$ and $e$ must be complemented and $f$ may not. Because each row in the $S$-boxes is a permutation and because $f$ may not be complemented in $S_{i}, a$ must be complemented in $S_{i}$. Remark that in fact one must still complement bit $c$ or $d$ in $S_{i+1}$. Indeed if only one input bit in an $S$-box is complemented, the output changes.

$\diamond$

We have now proven the theorem. It is now very easy to generate in a systematic way several examples for which the function $f$ remains constant even if some bits are complemented.

\subsection{The key clustering}

We analyze the clustering from the point of view that DES contains $\mathrm{j}$ rounds, where $j$ is between 1 and 16. The input for these $j$ rounds is fixed, while we complement or change some bits of the key. So if we speak now about an input of an $S$-box, this input is related to a modification of the key.

We first prove some general theorems for the key clustering, and afterwards we give some examples. 


\subsubsection{A general approach}

First of all for a fixed input the permutation $I P$ has no influence on the key clustering. We can start the analysis from $L_{s}$ and $R_{s}$. This means that if we are interested in a complete DES analysis $s=0$ and $j=16$. Let us now apply DES with the key $K$ and $K^{\prime}$ and call the subkeys $K_{1}$ till $K_{16}$ and $K_{1}^{\prime}$ till $K_{16}^{\prime}$. The key $K$ will produce some $L$ and $R$ register content, while $K^{\prime}$ produces $L^{\prime}$ and $R^{\prime}$. The effect of the first of the $j$ rounds is that in the case we use the key $K$ we have $L_{s+1}=R_{s}$ and $R_{s+1}=L_{s} \oplus f\left(R_{s}, K_{s+1}\right)$. Applying the key $K^{\prime}$ we obtain $L_{s+1}^{\prime}=R_{s}$ and $R_{s+1}^{\prime}=L_{s} \oplus f\left(R_{s}, K_{s+1}^{\prime}\right)$. After $t$ rounds we obtain using key $K$ the register contents $L_{s+t}=R_{s+t-1}$ and $R_{s+t}=L_{s+t-1} \oplus f\left(R_{s+t-1}, K_{s+t}\right)$. Using the key $K^{\prime}$ we have: $L_{s+t}^{\prime}=R_{s+t-1}^{\prime}$ and $R_{s+t}^{\prime}=L_{s+t-1}^{\prime} \oplus f\left(R_{s+t-1}^{\prime}, K_{s+t}^{\prime}\right)$. Remark that in general by changing the key the contents of the registers $L$ and $R$ change too. Let us now call $H_{s+t}=f\left(R_{s+t-1}, K_{s+t}\right) \oplus f\left(R_{s+t-1}^{\prime}, K_{s+t}^{\prime}\right)$. It is now easy to see using (Davio \& al., 1983) that the global effect of a change in the key has no final effect on the ciphertext if the two following conditions are satisfied together.

1. $H_{s+1} \oplus H_{s+3} \oplus H_{s+5} \oplus \ldots \oplus H_{t}=0$, where $t=s+j$ if $j$ is odd, else $t=s+j-1$.

2. $H_{s+2} \oplus H_{s+4} \oplus H_{s+6} \oplus \ldots \oplus H_{u}=0$, where $u=s+j$ if $j$ is even, else $u=s+j-1$.

Using previous conditions it is now easy to analyze the conditions necessary for key clustering if one analyzes only 1, 2, 3 or 4 rounds. The analyze of more rounds seems to be more difficult if one want to have a complete analysis.

\subsubsection{An analysis of the key clustering in a DES with 1 or 2,3 or 4 rounds}

In the case 1 round is considered we must have $B_{s+1}=0$. This means $f\left(R_{s}, K_{s+1}\right)=f\left(R_{s}, K_{s+1}^{\prime}\right)$. Using previous knowledge on the $S$-boxes this means that the input of an $S$-box is not changed or that at least two bits change. It is very easy to generate several examples for this case. Using the fact that $E$ is an expansion of 32 bits to 48 bits and its structure (Davio $\&$ al., 1983) and because $P C_{2}$ selects only 48 bits out of the 56 bits of the key we have the following result. For each (cleartext,ciphertext) pair in a one round DES there exist exactly $2^{24}$ keys which generate the same (cleartext, ciphertext) pair starting from a fixed cleartext. If a similar remark remains true for the complete DES algorithm (16 rounds), DES is very easy to break using a simplified exhaustive attack. Let us therefore start to analyze more rounds.

In the case 2 rounds are considered we must have $H_{s+1}=0$ and $H_{s+2}=0$. This means $f\left(R_{s}, K_{s+1}\right)=f\left(R_{s}, K_{s+1}^{\prime}\right)$, as in previous case, and additionally $f\left(R_{s+1}, K_{s+1}\right)=f\left(R_{s+1}, K_{s+1}^{\prime}\right)$, because from the first equality we have $R_{s+1}^{\prime}=$ 
$R_{s+1}$. Remark that the $S$-boxes must satisfy similar conditions as in the case only one round was considered. However to satisfy it for the two rounds together we must take the key scheduling in DES into consideration. We now give a simple example of it.

Example 1. If one complements the bits 3 and 44 (in the NBS notation) of any 64 bit key, then there exists $6.2^{59}$ pairs of (cleartext, ciphertext) which remain identical during round 1 and 2 in DES. In other words, $1 / 5$ of all pairs (cleartext, ciphertext) are not affected by the complementation of 2 bits of the key, during round 1 and 2 .

Let us now explain what happens and how one can calculate the (cleartext,ciphertext) pairs. The bits 3 and 44 go both after the key scheduling in the first round to $S_{3}$ and become there the inputs $a$ and $e$. We can verify that for 6 out of 32 (or 12 out of 64) possible inputs a complementation of $a$ and $e$ in $S_{3}$ does not change the output. This means that the possible inputs for which the above property is true are restricted from $2^{64}$ to $6.2^{59}$. The cardinality of the set of cleartext for which the explained clustering is satisfied is independent of the used key. However the set of cleartext for which the above clusering changes if other keys are considered. This is a consequence of the structure in the function $f$. Now we must still analyze which restrictions the second round imposes on the possible cleartext. The analysis in this example is straightforward because the key bits 3 and 44 are not selected in the second round, so no extra condition is necessary.

One may observe that we were lucky in the construction of the previous example. First the non-selection of the key bits in the second iteration seems to be lucky. Secondly example 1 is only valid for rounds 1 and 2 in DES. In the following example the reader can observe that similar examples can be given for all rounds and that the non-selection of some key bits is not necessary.

Example 2. This example is true for most consecutive rounds. As a consequence of the ideas of Neutjens on the key scheduling (see section 3.4), two consecutive rounds can mostly be analyzed systematically (Neutjens, 1983). This is true if one uses two shifts in the key scheduling, as represented by the NBS, to move to the next round. This means the rounds $2-3,3-4,4-5,5-6,6-7,7-8$, 9-10,10-11,11-12,12-13,13-14 and 14-15. In order not to affect the generality we will use a more general descriptions of the property. If one complements the two bits of the key which will "arrive" in $S$-box 4 at locations $a$ and $e$ during the first of the two above rounds, then for every key there exists $36.2^{54}$ (or about $1 / 29$ of all possible) pairs (cleartext, ciphertext) which remain identical during two consecutive rounds mentioned earlier. This can be analyzed using the ideas of Neutjens on the key scheduling (Neutjens, 1983) and using our properties of the $S$-boxes.

Let us now consider three consecutive rounds. First more restrictions on the 
cleartext are then imposed in order not to affect the ciphertext if one modify the key. This is a consequence of the key scheduling. However the output of the function $f$ in the first and last (of the three) rounds must no longer be constant (see section 3.6.1). This relaxes the imposed restrictions. Let us give a short example to illustrate it. Remark the authors have yet generalized this example, but this is out of the scope of this paper.

Example 3 The three consecutive rounds may be 2-3-4, 3-4-5, 4-5-6, 5-6-7, 67-8, 9-10-11, 10-11-12, 11-12-13, 12-13-14 and 13-14-15. Hereto one complements (e.g.) three bits of the key. In our example the key bits must "arrive" at location $a$ and $d$ in $S$-box 8 in the first round (of the three consecutive) and at location $d$ in $S$-box 4. We impose the extra condition that bit 15 of the output of $f$ (after the box $P$ ) must be complemented in the first and last round as consequence of the modification of the key. We can then analyze that for $50 \%$ of the keys and for 1 on 1024 cleartext, the ciphertext is not modified. For the other $50 \%$ of the keys this happens for 1 on 4096 cleartexts.

\section{Conclusions and perspectives}

A cryptographic system can only be considered secure if a small modification in the cleartext and/or in the key strongly affect on a non-linear way the ciphertext. We described techniques for analyzing this constraint for DES. We found that if DES had only a few rounds it would be a bad system. Our analysis demonstrated at the same time that the known probalistic test done on DES are insufficient to conclude that the scheme is secure. Were it possible to work out on a 16-round DES the techniques presented here one could possibly prove the so often alleged existence of a key clustering in DES. 


\section{References}

[1] ANSI X3.92-1981, "Data Encryption Algorithm," American National Standards Institute, New York (December 31, 1980).

[2] R. Bernhard, "Breaching system security," Spectrum, vol. 19, pp. 24-31 (1982).

[3] M. Davio, Y. Desmedt, M. Fosseprez, R. Govaerts, J. Hulsbosch, P. Neutjens, P. Piret, J.-J. Quisquater, J. Vandewalle \& P. Wouters, "Analytical characteristics of the DES," pp. 171-202, in Adrance in cryptology: Proceedings of Crypto 83, ed. D. L. Chaum, Plenum Press, New York (1984).

[4] FIPS publication 46, "Data Encryption Standard," Federal Information Processing Standard, National Boreau of Standards, U.S. Department of Commerce, Washington, D.C. (January 1977).

[5] FIPS publication 81, "DES modes of operation," Federal Information Processing Standard, National Bureau of Standards, U.S. Department of Commerce, Washington, D.C. $(1980)$.

[6] M. E. Hellman, R. Merkle, R. Schroeppel, L. Washington, W. Diffie, S. Pohlig \& P. Schweitzer, "Results of an initial attempt to cryptanalyze the NBS data encryption standard," SEL 76-042, Stanford University (1976).

[7] ISO/DP 8227 (Draft proposal), "Data encipherment, specification of algorithm DEA1," (1983).

[8] A G. Konheim, Cryptography: A primer, J. Wiley, New York (1981).

[0] C. H. Meyer \& S. M. Matyas, Cryptography: A new dimension in computer data security, J. Wiley, New York (1982).

[10] R. Morris, N. J. A. Sloane \& A D Wyner, "Assessment of the NBS proposed Data Encryption Standard," Cryptologia, vol. 1, pp. 301-306 (1977).

[11] P. Neutjens, "Diepere inzichten en eenvoudige hardware voor DES cryptografisch algoritme aan de hand van equivalente strukturen," Final work, Katholieke Universiteit Leuven, Belgium (1983). 